\title{
A Prospective Evaluation of Acute Cerebral Ischemic Stroke by Conventional and Diffusion-weighted Magnetic Resonance Imaging Sequences
}

\author{
${ }^{1}$ Arvinder Singh, ${ }^{2}$ Mandeep Singh, ${ }^{3}$ Gurinder Bir Mahal, ${ }^{4}$ Ramesh Chander
}

\begin{abstract}
Aim: The aim of this study is to compare the diffusion-weighted magnetic resonance imaging (DW-MRI) with conventional MRI in the evaluation of acute cerebral ischemic stroke.

Materials and methods: In this prospective study, for 2 years, 50 patients who were clinically suspected cases of acute ischemic stroke (AIS) underwent MRI using a 1.5 tesla scanner (SIEMEN MAGNETOM Aera) in the Department of Radiodiagnosis, Guru Nanak Dev Medical College, Amritsar, Punjab. The patients from all age groups and both sexes were included in this study.

Results: In our study of 50 cases, the age range of patients was from 2 years to 80 years with a majority of $40(90 \%)$ patients above 40 years age with $32(64 \%)$ males and $18(36 \%)$ females. About 20 (40\%) patients presented with weakness/numbness on either side. The conventional MRI had a sensitivity of $50 \%$ in detecting stroke in patients presented within 3 hours of the onset of symptoms, while DW-MRI had a sensitivity of $100 \%$. For the patients presented within 3 to 6 hours, conventional MRI and DWI had a sensitivity of $66 \%$ and $100 \%$; for the patients presented within 6 to 12 hours, conventional MRI and DWI had a sensitivity of $85 \%$ and $100 \%$, and for patients presented within 12 to 24 hours, conventional MRI and DWI had a sensitivity of $92.3 \%$ and $100 \%$, respectively. The middle cerebral artery (MCA) was the most commonly involved territory.

Conclusion: The DW sequence is the sensitive modality in the diagnostic evaluation of acute stroke as compared to conventional MRI sequences. It plays a pivotal role in the localization and characterization of acute ischemic lesions and, hence, effective and early treatment with thrombolytic therapy is needed.
\end{abstract}

Keywords: Acute ischemic stroke, Diffusion-weighted image, $\mathrm{T} 1$ weighted (T1W), T2 weighted (T2W).

How to cite this article: Singh A, Singh M, Mahal GB, Chander R. AProspective Evaluation of Acute Cerebral Ischemic Stroke by Conventional and Diffusion-weighted Magnetic Resonance Imaging Sequences, Curr Trends in Diagn Treat 2018;2(2):77-81.

Source of support: Nil

Conflict of interest: None

${ }^{1}$ Professor, ${ }^{2}$ Junior Resident, ${ }^{3}$ Associate Professor, ${ }^{4}$ Professor and Head

${ }^{1-4}$ Department of Radiodiagnosis, Government Medical College, Amritsar, Punjab, India

Corresponding Author: Mandeep Singh, Junior Resident, Department of Radiodiagnosis, Government Medical College, Amritsar, Punjab, India, e-mail: arvinderdr@rediffmail.com

\section{INTRODUCTION}

The current World Health Organization definition of stroke (introduced in 1970 and still used) is a rapidly developing clinical signs of focal (or global) disturbance of cerebral function, lasting more than 24 hours or leading to death, with no apparent cause other than that of vascular origin. ${ }^{1}$

Transient ischemic attacks are episodes of temporary and focal dysfunction of vascular origin, which are variable in duration, commonly lasting from 2 to 15 minutes, but occasionally lasting as long as a day ( 24 hours). They leave no persistent neurological deficit. ${ }^{2}$

Cerebral ischemia can be defined as the diminution of cerebral blood flow to all or a portion of the brain, below the level needed to maintain normal cerebral function. The diagnosis, localization, and characterization of acute ischemic brain lesions have become increasingly important following the advent of thrombolytic therapy in the management of patients with AIS. Restoration of function within this ischemic penumbra represents the target of early stroke strategies. It has been shown that intravenous recombinant tissue plasminogen activator (t-PA) when administered within 3 hours of the onset of stroke results in an improved long-term clinical outcome. ${ }^{3,4}$

A stroke may be classified as early hyperacute stroke, within 0 to 6 hours; late hyperacute stroke, within 6 to 24 hours; acute stroke, within 24 hours to 7 days; subacute stroke, between 1 and 3 weeks; and chronic stroke, more than 3 weeks old. ${ }^{5}$

The diffusion-weighted image (DWI) can be obtained within 10 minutes at some centers and dramatically alters care because the clinical determination of ischemic stroke can be confirmed quickly. DWI is used to detect early ischemic changes (acute stroke, early ischemic change, and cytotoxic edema) with greater conspicuity than a standard MRI. MRI with diffusion is quickly becoming the gold standard in acute stroke imaging, once a hemorrhagic stroke has been excluded. ${ }^{6,7}$

\section{AIMS AND OBJECTIVES}

- To compare the role of DW-MRI with that of conventional MRI techniques in patients presenting within 
24 hours of the onset of symptoms of acute cerebral ischemia.

- To compare the sensitivity and specificity of conventional MRI with that of DW-MRI in acute cerebral ischemic stroke patients.

\section{MATERIALS AND METHODS}

The prospective study was conducted in the Department of Radiodiagnosis, Guru Nanak Dev Medical College \& Hospital, Amritsar, Punjab, with the permission from the Institutional Ethics Committee, Govt. Medical College, Amritsar. Fifty patients with signs and symptoms of cerebral ischemia, presenting within 24 hours of onset will be included in the study after taking written informed consent from them/guardian.

MRI was performed with a Siemens Aera 1.5 tesla system with echo-planar capability using a standard protocol for conventional MRI, including T1, T2, Flair, and DWI. In our study, the patients presented for investigation within 3 hours, 3 to 6 hours, between 6 and 12 hours, and within 12 to 24 hours of the onset of signs and symptoms were studied.

The patients presenting with the onset of signs and symptoms after 24 hours, having intracranial hemorrhage, history of head injury associated with an acute neurological deficit, seizures, a diagnosed case of intracranial tumor or abscess, and MRI findings suggestive of demyelinating disorders were excluded from the study, which was done for a duration of about 2 years.

An appropriate statistical analysis was performed to evaluate the sensitivity of each of the imaging sequences in detecting the presence or absence of cerebral ischemia. The final diagnosis of the patient was based on the clinical picture and subsequent MRI was taken as a collective gold standard against which various sequences were evaluated.

Statistical analysis in the form of mean \pm SD value and $p$ value was performed.

\section{OBSERVATIONS}

The age range of patients was from 2 years to 80 years with a majority of $25(50 \%)$ patients in the age range of

Table 1: Age-wise distribution of the patients with stroke

\begin{tabular}{llc}
\multicolumn{3}{c}{$(n=50)$} \\
\hline Age group (in years) & No. of cases & Percentage (\%) \\
\hline$<20$ & 2 & 4.0 \\
$21-40$ & 3 & 6.0 \\
$41-60$ & 25 & 50.0 \\
$61-80$ & 18 & 36.0 \\
$>80$ & 2 & 4.0 \\
Total & 50 & 100.0 \\
Mean \pm SD & $57.35 \pm 15.73$ & \\
\hline
\end{tabular}

Table 2: Gender-wise distribution of patients with stroke $(n=50)$

\begin{tabular}{llc}
\hline Gender & No. of cases & Percentage \\
\hline Male & 32 & 64.0 \\
Female & 18 & 36.0 \\
Total & 50 & 100.0 \\
\hline
\end{tabular}

Table 3: Duration of signs and symptoms of patients with stroke $(n=50)$

\begin{tabular}{lcc}
\hline Duration (in hours) & No. of cases & Percentage \\
\hline$<3$ & 8 & 16.0 \\
$3-6$ & 9 & 18.0 \\
$6-12$ & 20 & 40.0 \\
$12-24$ & 13 & 26.0 \\
Total & 50 & 100.0 \\
\hline
\end{tabular}

41 to 60 years, followed by 18 (36\%) patients between 61 and 80 years of age as shown in Table 1 .

In our study of 50 cases, there were a majority of 32 $(64 \%)$ males with $18(36 \%)$ females with a male-to-female ratio of 1.7:1 as shown in Table 2.

Table 3 shows that a majority of $20(40 \%)$ patients presented in the hospital between 6 and 12 hours of the symptoms, followed by 13 (26\%) patients by 12 to 24 hours, $9(18 \%)$ patients between 3 and 6 hours, and only $8(16 \%)$ patients presented within 3 hours of the onset of symptoms.

The MCA was the most commonly involved intracerebral arterial territory in $41(82 \%)$ cases, with the left MCA territory involved in $25(50 \%)$ cases and the right MCA involved in 16 (32\%) cases. The PICA was the next commonly involved cerebral artery in four $(8 \%)$ cases followed by anterior and posterior cerebral arteries in two $(4 \%)$ cases each. The anterior cerebral artery was involved in only one (2\%) case as shown in Table 4.

The conventional MRI had a sensitivity of $50 \%$ in detecting stroke in patients presenting within 0 to 3 hours of the onset of symptoms, while DWI had a sensitivity of about $100 \%$. For patients who presented within 3 to 6 hours, the conventional MRI had a sensitivity of $66 \%$ and DWI had a sensitivity of $100 \%$. For patients who presented within 6 to 12 hours, the conventional MRI had a sensitivity of $85 \%$ and DWI had a sensitivity of

Table 4: Involvement of cerebral arterial territories in patients with stroke $(n=50)$

\begin{tabular}{|c|c|c|c|}
\hline \multicolumn{2}{|c|}{ Territory involved } & \multirow{2}{*}{$\begin{array}{l}\text { No. of cases } \\
25\end{array}$} & \multirow{2}{*}{$\frac{\text { Percentage }}{50.0}$} \\
\hline Middle & Left MCA & & \\
\hline Cerebral & Right MCA & 16 & 32.0 \\
\hline \multicolumn{4}{|l|}{ Artery } \\
\hline \multicolumn{2}{|c|}{ Left anterior cerebral artery } & 2 & 4.0 \\
\hline \multicolumn{2}{|c|}{ Left posterior cerebral artery } & 2 & 4.0 \\
\hline \multicolumn{2}{|c|}{$\begin{array}{l}\text { Left posterior inferior cerebellar } \\
\text { artery }\end{array}$} & 4 & 8.0 \\
\hline \multicolumn{2}{|c|}{ Right anterior cerebral artery } & 1 & 2.0 \\
\hline \multicolumn{2}{|l|}{ Total } & 50 & 100.0 \\
\hline
\end{tabular}




\begin{tabular}{|c|c|c|c|c|}
\hline \multicolumn{5}{|c|}{$\begin{array}{l}\text { Table 5: Comparison of the sensitivity of conventional MRI } \\
\text { and DWI in detecting acute cerebral ischemia in patients } \\
\text { presented within } 0 \text { to } 3 \text { hours, } 3 \text { to } 6 \text { hours, } 6 \text { to } 12 \text { hours, and } \\
12 \text { to } 24 \text { hours of the onset of signs/symptoms }\end{array}$} \\
\hline MRI sequence & $\begin{array}{l}0-3 \\
\text { hours }\end{array}$ & $\begin{array}{l}3-6 \\
\text { hours }\end{array}$ & $\begin{array}{l}6-12 \\
\text { hours }\end{array}$ & $\begin{array}{l}12-24 \\
\text { hours }\end{array}$ \\
\hline DWI & $100 \%$ & $100 \%$ & $100 \%$ & $100 \%$ \\
\hline Conventional MRI & $50 \%$ & $66.6 \%$ & $85 \%$ & $92.3 \%$ \\
\hline$p$ value & 0.007 & 0.009 & 0.035 & 0.141 \\
\hline
\end{tabular}

$100 \%$ and for patients presented within 12 to 24 hours, the conventional MRI had a sensitivity of $92.3 \%$ and DWI had a sensitivity of $100 \%$ as shown in Table 5 .

\section{DISCUSSION}

AIS is the leading cause of morbidity worldwide and is the leading cause of mortality. ${ }^{8}$ The approval of IV-rt-PA was a landmark step toward the treatment of AIS when given between a window period of 3 and 4.5 hours of the onset of initial symptoms. The delay in imaging and achieving reperfusion is the cause of severe morbidity and mortality with a poor outcome. ${ }^{9}$

Therefore, the present study was carried out on 50 patients who presented with the clinical diagnosis of AIS, for the evaluation with MRI. The sensitivity of detecting ischemic areas in these patients was calculated with both conventional MRI and DWI sequences.

The study on 50 patients showed that the maximum number of patients who presented with signs and symptoms of AIS was above 40 years old. This indicates that as the age advances, there was an increase in the incidence of stroke (Table 1). The mean age of the patients in our study was about 59 years with an average age of 56.7 years in males and 62.4 years in females. This is similar to the findings of the study done by Roger et al. which showed that the incidence of stroke rapidly increases with age, doubling for each decade after the age of 55 years. ${ }^{10}$

In our present study of 50 patients, 32 (64\%) patients were males and $18(36 \%)$ patients were females with a male-to-female ratio of 1.7:1 (Table 2). It is well accepted that the age is one of the most important factors and that the incidence of stroke is higher in men than in women. This is in concordance with the findings of epidemiological study conducted by Appelros Peter et al. in a Western European journal, which showed that stroke was more common in men than in women. The male stroke incidence rate was $33 \%$ higher and the stroke prevalence was $41 \%$ higher than in females. ${ }^{11}$ This is also in concordance with the studies done by Reeves et al. and Nagaraja et al., which showed that age-specific stroke incidence rates were higher in men than in women. ${ }^{12,13}$

In our study of 50 patients, $8(16 \%)$ patients presented in the hospital for investigation within 3 hours, 9 (18\%) patients within 3 to 6 hours, a majority of $20(40 \%)$ patients presented between 6 and 12 hours, and $13(26 \%)$ patients presented within 12 to 24 hours of the onset of signs and symptoms. Since the therapeutic window for an effective thrombolytic therapy is within 3 hours and in selected patients within 6 hours, the time of presentation is important in the management of patients presenting with symptoms of acute cerebral ischemic stroke. These findings are similar to the study done by Lees et al., which showed that intravenous (IV) administration of alteplase is the only US Food and Drug Administration (FDA)-approved medical therapy for the treatment of patients with AIS. Its use is associated with improved outcomes for a broad group of patients. The earlier treatment is more likely to give a favorable outcome. ${ }^{14}$ This is also in accordance with the study conducted at the National Institute of Neurological Disorders and Stroke (NINDS) study. ${ }^{15}$ A similar outcome was shown by the study conducted at the National Institutes of Health Stroke Scale (NIHSS). There was a significantly better outcome among those treated with IV alteplase. The alteplase-treated patients had a $30 \%$ higher probability of recovering with little or no deficit after 3 months. ${ }^{16}$

In our study of 50 cases, the MCA was the most commonly involved intracerebral arterial territory. The left MCA territory was involved in $25(50 \%)$ patients and the right MCA being involved in 16 (32\%) patients. This is similar to the study done by Paciaroni et al. where a correlation between the potential causes of stroke (TOAST etiological groups) and the involvement of different vascular territories is seen. They found that main MCA territory was involved in $(40.9 \%)$, with perforating branches of MCA (35.7\%), and watershed infarcts (68.2\%) patients. $^{17}$

Among the patients who presented within 3 hours of sign and symptoms, there was a marked difference in the sensitivity with a conventional MRI showing a sensitivity of $50 \%$ and DWI showing a sensitivity of $100 \%$. For the patients presented between 3 and 6 hours of the onset of symptoms, the conventional MRI had a sensitivity of $66.6 \%$ and DWI had a sensitivity of $100 \%$. This showed that there is a substantial advantage of DWI over conventional MRI in the sensitivity of detecting the early stroke, even more pronounced, within 6 hours of the onset of symptoms.

The findings in this study support the conclusions of previous studies that DWI is superior to conventional MRI in detecting stroke within 24 hours of presentation. This is similar to the cross-sectional study conducted by Nisar et al. in the Department of Radiology, Pakistan Institute of Medical Sciences, Islamabad, in which DWI had a sensitivity of $98 \%$, a specificity of $100 \%$, a positive 
predictive value of $100 \%$, and a negative predictive value of $67 \%$. This concluded that DW-MRI of the brain is highly accurate in the diagnosis of an acute stroke. ${ }^{18,19}$

The similar findings were shown by the studies done by Merino et al. and Warach et al., which showed that the sensitivity of DWI was 88 to $100 \%$ with a specificity of 95 to $100 \%$. They also confirmed in their studies that DWI can detect ischemic areas, even within 3 minutes of stroke onset. ${ }^{20,21}$

In our study of 50 patients, conventional MRI had a sensitivity of $85 \%$ in 6 to 12 hours and $92.3 \%$ in 12 to 24 hours of the onset of signs and symptoms, while DWI had a sensitivity of $100 \%$ in 12 to 24 hours of the onset of symptoms. Similar findings were seen in the study done by O'Brien et al., which showed that high signal intensity is not usually seen in T2-weighted imaging until at least 8 hours after the initial ischemic insult. It persists into the chronic phase and usually maximizes in the subacute phase. The time at which T2-weighted imaging findings become positive varies; they found that it is likely to be longer than 8 hours. ${ }^{22}$ The findings also support the studies done by Nah et al. and Aoki et al., which showed that hyperintense signals on $\mathrm{T} 2$ and FLAIR sequences, indicates that the ischemia is likely to be more than 8 hours. So, conventional MRI sequences may help in classifying the subtype of index stroke into subacute or chronic ischemic lesions. ${ }^{23,24}$

The DWI sequences are superior to the conventional MRI in detecting stroke within 24 hours of the onset of symptoms. The conventional MRI had a sensitivity of $50 \%$ within 0 to 3 hours and $66.66 \%$ in patients presented within 3 to 6 hours, respectively, after the onset of symptoms, while DWI had a sensitivity of $100 \%$. A hyperintense signal on conventional MR sequences indicates that ischemia is not likely less than 6 to 8 hours, which is the potential contraindication of intravenous thrombolytic therapy. The high sensitivity in detecting early cerebral ischemia has placed DWI along with conventional MRI firmly as an indispensable tool in the initial assessment of patients with signs and symptoms of acute cerebral ischemia, especially in identifying a potential candidate for thrombolytic therapy.

\section{CONCLUSION}

Stroke is a medical emergency and a prompt treatment is crucial to minimize brain damage and potential complications. The patients with AIS are at an increased risk of all causes of mortality and morbidity. Ideally, the therapy with a potent clot-busting drug should be given within 3 hours. In some instances, t-PA can be given up to 4.5 hours after stroke symptoms and sooner. The sensitivity of DWI is 100\% within 3 hours of the onset of symptoms in comparison to that of conventional MRI, which is $50 \%$. The sensitivity of conventional MRI and DWI within 3 to 6 hours is about $66.66 \%$ and $100 \%$, respectively. On conventional MRI (T2-weighted and FLAIR images), ischemic infarction appears as a hyperintense lesion usually seen within the first 8 to 12 hours after the onset of stroke symptoms. So, conventional MRI helps in classifying the subtype of stroke, i.e., subacute or chronic ischemic lesions.

\section{REFERENCES}

1. Aho $\mathrm{K}$, Harmsen $\mathrm{P}, \mathrm{Hatano} \mathrm{S}$ et al. Cerebrovascular disease in the community: results of a WHO collaborative study. Bull World Health Organ 1980;58(1):113-330.

2. A classification and outline of cerebrovascular diseases. II. Stroke 1975;6(5):564-516.

3. Kasmaei HD, Baratloo A, Nasiri Z et al. Recombinant Tissue Plasminogen Activator Administration in Patients with Cerebrovascular Accident a case series. Archives of Neuroscience 2015;2(2):23315-23318.

4. Lin MP, Liebeskind DS. Imaging of ischemic stroke. Continuum (Minneap Minn) Neuroimaging 2016;22(5):1399-1423.

5. Allen LM, Hasso AN, Handwerker J et al. Sequence-specific MR Imaging Findings That Are Useful in Dating Ischemic Stroke. Radio Graphics 2012;32(5):1285-1297.

6. Kunz A, Hahn G, Mucha D et al. Echo-enhanced transcranial color-coded duplex sonography in the diagnosis of cerebrovascular events: a validation study. AJNR Amj Neuroradiol 2006;27(10):2122-2127.

7. McGraw-Hill. Emergency Radiology: Case Studies Access Emergency Medicine. Medicalbook 2008;8:434-461.

8. Khandelwal P, Yavagal DR, Sacco RL. Acute ischemic stroke Intervention. Journal of the American College of Cardiology 2016;67(22):2631-2644.

9. Del Zappo GJ, Saver JL, Jauch EC. For the American Heart Associate statistics committee and stroke statistics subcommittee. Expansion of the time window for treatment of acute ischemic stroke with intravenous tissue plasminogen activator a science advisory from the American Heart Association/ American Stroke Association. Stroke 2009;40(8):2945-2948.

10. Roger VL, Go AS, Lloyd-Jones DM et al. American Heart Association Statistics Committee and Stroke Statistics Subcommittee. 2011;123(4):18-19.

11. Appelros P, Stegmayr B, Teréntm A. Sex differences in stroke epidemiology a systematic review stroke. 2009;40(4):1082-1089.

12. Reeves MJ, Bushnell CD, Howard G et al. Sex differences in stroke: epidemiology, clinical presentation, medical care, and outcomes. Lancet Neurol 2008;7(10):915-926.

13. Nagaraja D, Gururaj G, Girish N et al. Feasibility study of stroke surveillance data from Bangalore, India. Indian J Med Res 2009;130(4):396-403.

14. Lees KR, Bluhmki E, von Kummer R et al. Time to treatment with intravenous alteplase and outcome in stroke: an updated pooled analysis of ECASS, ATLANTIS, NINDS, and EPITHET trials. Lancet 2010;375(9727):1695-1703.

15. The National Institute of Neurological Disorders and Stroke rt-PA Stroke Study Group. Tissue plasminogen activator for Acute ischemic stroke. N Engl J Med 1995;333(24):1581-1587.

16. Generalized efficacy of t-PA for acute stroke. Subgroup analysis of the NINDS t-PA Stroke Trial Stroke.1997;28(11):2119-2125. 
17. Paciaroni M, Silvestrelli G, Caso V et al. Neurovascular territory involved in different etiological subtypes of ischemic stroke in the Perugia Stroke Registry. Eur J Neurol 2003;10(4):361-365.

18. Nisar S, Nizami AA, Khan SF. Accuracy of diffusion-weighted magnetic resonance imaging in the detection of acute cerebral infarcts. P J M H S 2014:(8):832-836.

19. Rosso C, Drier A, Lacroix D et al. Diffusion-weighted MRI in acute stroke within the first 6 hours Neurology. 2010;74(24):1946-1953.

20. Warach S, Gaa J, Siewert B et al. Acute human stroke studied by whole brain echo planar diffusion-weighted magnetic resonance imaging. Ann Neurol 1995;37(2):231-241.
21. Merino JG, Warach S. Imaging of acute stroke. Nat Rev Neurol 2010;6(10):560-571.

22. O'Brien P, Sellar RJ, Wardlaw JM. Fogging on T2-weighted MR after acute ischaemic stroke: how often might this occur and what are the implications. Neuroradiology 2004;46(8):635-641.

23. Nah HW, Kang DW, Kwon SU et al. Diversity of single small subcortical infarctions according to infarct location and parent artery disease: analysis of indicators for small vessel disease and atherosclerosis. Stroke 2010;41(12):2822-2827.

24. Aoki J, Kimura K, Iguchi $Y$ et al. FLAIR can estimate the onset time in acute ischemic stroke patients. J Neurol Sci 2010;293(1-2):39-44. 